\title{
An overview of the low temperature microgravity physics facility capabilities
}

\author{
J.F. Pensinger, A.P. Croonquist, F.C. Liu, M.E. Larson, and T.C. Chui \\ Jet Propulsion Laboratory, California Institute of Technology \\ Pasadena, California 91109, USA \\ E-mail: john.f.pensinger@jpl.nasa.gov
}

Received December 19, 2002

\begin{abstract}
The Low Temperature Microgravity Physics Facility currently in the design phase is a multiple user and multiple flight facility intended to provide a long duration low temperature environment onboard the International Space Station. The Facility will provide a unique platform for scientific investigations requiring both low temperature and microgravity conditions. It will be attached to the Japanese Experiment Module's Exposed Facility and can house two science instruments each flight. The Facility consists of a 180-liter superfluid helium dewar, a support enclosure, and control electronics. The facility will be launched full of cryogen, and retrieved after the cryogen is depleted. Industrial partners are responsible for building the reusable facility and standard parts of the instruments. Principal Investigators from universities and other institutions are contracted to develop major parts of the science instrument package. An overview of the detailed technical capabilities of the facility will be presented in this paper.
\end{abstract}

PACS: 64.60.- $\mathbf{i}$

\section{Introduction}

The Low Temperature Microgravity Physics Facility (LTMPF) [1] is the next step in a series of three very successful space flight low temperature experiments - The Superfluid Helium Experiment [2] (1985), the Lambda Point Experiment [3] (1992), and the Confined Helium Experiment [4] (1997). This series of experiments have proven that very high-resolution experimentation can be implemented in the hostile environment of space. For example, it was shown that temperature can be measured to better than $0.1 \mathrm{nK}$ in space using Superconducting Quantum Interference Device (SQUID) magnetometers [4]. These high-resolution components, which require operating at low temperatures, when combined with the microgravity condition due to the International Space Station's (ISS) orbit, open the door to many exciting new investigations with the potential of making breakthrough findings. The LTMPF is designed to broaden investigation opportunities requiring these high-resolution capabilities in Space. Science selection is done through a NASA Research Announcement (NRA) periodically to select investigations to fly on each mission. Already, there are six investigations planned for the first two flights, but these investigations are not discussed in this paper. To meet the demands of the science community, the Jet Propulsion Laboratory has partnered with Ball Aerospace and Technologies Corporation (BATC) to build the dewar and the facility enclosure. Design-Net Engineering has been selected to develop the electronics and software, and Swales Aerospace has been selected to help build the instrument thermal mechanical structure. In the following, we will describe an overview of the design and technical capabilities of the facility.

\section{System design and capabilities}

Figure 1 below shows the LTMPF. It consists of the dewar, the electronics, the radiators, the science instruments which mount inside the dewar, and the facility enclosure that houses the dewar, the electronics and various interface components.

These interface components will be provided to LTMPF by ISS and NASA. They consist of the Grapple Fixture for the Japanese Experiment Module's (JEM) robotic arm to hold onto; the Flight Releasable Attachment Mechanism (FRAM) for attach- 


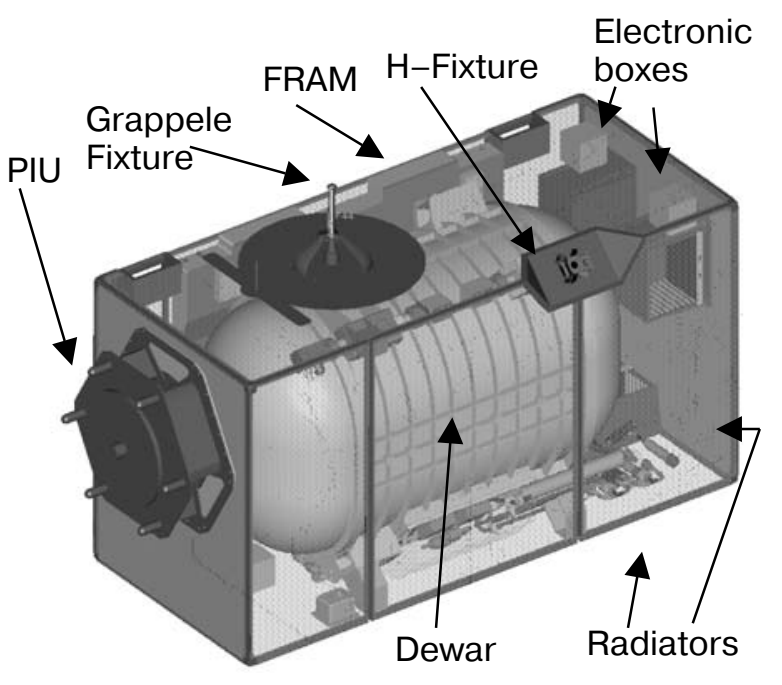

Fig. 1. The Low Temperature Microgravity Physics Facility overview.

ing to the launch carrier and the ISS robotic arm, and the Payload Interface Unit (PIU) for attaching to the JEM-EF and accessing the $120 \mathrm{~V}$ dc electrical power and the communication interfaces. The first of three VME chassis is used to control the dewar and to communicate with the ISS; the other two electronics assemblies are used to control experiments in the two cryogenic inserts. Tools on the end of the space station robotic arm also interface with the $H$-Fixture for transferring the payload to and from the Shuttle. The maximum power available to the electronics during the mission is limited by the ability of the radiators to reject heat of about $235 \mathrm{~W}$. Uplink low rate commands of no more than $864 \mathrm{bits} / \mathrm{s}$ are provided for the payload through a 1553B interface, and the highspeed downlink telemetry for the data is capable of handling 1.5 Mbps using Ethernet. Total mass of the LTMPF is limited to $600 \mathrm{~kg}$ and the envelope size is $1.85 \mathrm{~m}$ by $0.816 \mathrm{~m}$ by $1.037 \mathrm{~m}$.

\section{Dewar design and capabilities}

The dewar contains approximately 180 liters of liquid helium and is expected to last about 4.5 months on orbit. The cross section is not cylindrical, but oblong in shape to maximize the use of the available volume within the Standard Japanese Experiment Module's Exposed Facility (JEM-EF) envelope. This large volume also helps the dewar to stay below the superfluid transition temperature, without active evacuation, for 112 hours prior to launch, so that full science time can be achieved if launched within the first 2 attempts. If launched at the third and later attempts the on-orbit science time will be reduced. The dewar is designed to operate at a base temperature of $1.6 \mathrm{~K}$. It has two $20 \mathrm{~cm}$ diameter openings, one on each end, to allow two separate science instruments to be mounted. Shielded low conductivity wires are routed through the dewar vacuum space, while high current leads and coaxial cables are routed through the vent line tubing to allow for better heat exchange with the vent gas. The superfluid in the dewar permits the maintenance of a heat sink $(<8 \mathrm{~mW})$ at a stable temperature $\sim(1.7 \pm 0.05) \mathrm{K}$ over many months.

\section{Enclosure design and capabilities}

The dewar's Vacuum Shell is the primary structural component of the LTMPF system. The electronics boxes and JEM-EF interface are mounted inside aluminum honeycomb panels that form a rectangular box around the system. The nadir-, wake, and part of the zenith-facing surfaces are thermal radiators while blankets cover the remaining panels. A separate honeycomb panel is used to support the releasable mechanism (FRAM) that attaches LTMPF to its launch carrier. These panels and the thermal design are being provided by BATC.

\section{Electronics capabilities}

There are three main electronic assemblies each housed in a separate VME box with its own single board computer. The Facility Electronic Assembly controls the function of the dewar and handles power and communication with the JEM-EF. It provides housekeeping data on various parts of the dewar and controls the vent valves of the two instruments. Small heat pulses can be used to estimate the amount of helium remaining. In addition, it provides environmental data on charged particle radiation and acceleration by interfacing with an onboard charged particle monitor and a Space Acceleration Measurement System (SAMS) accelerometer provided by Glen Research Center.

The two Experiment Electronic Assemblies each control one science instrument. The standard boards support resistance measurements of germanium elements to determine the temperature; low-, high-, and very high-precision heaters; high resolution measurements over extremely large dynamic ranges are made using dc superconducting quantum interference device magnetometers. Capability for relatively coarse temperature control is provided using germanium resistance thermometers and microwatt heater elements. For more precise control at and near the science cell a paramagnetic salt pill coupled to a SQUID with a high-precision heater can control the temperature to better than $1 \mathrm{nK}$. There are spare slots for Investigator's custom electronics boards unique to their experiment. All boards must conform to the VME 64 stan- 
dard with a $6 \mathrm{U}$ form factor and run with VxWork real time operating system.

The Facility electronics also include SQUID preamplifier/controller boxes that on one side interface with the high-resolution sensors inside the dewar and on the other a digital interface to a board in a VME chassis. On ascent a pressure-sensitive device or baroswitch and a battery pack are used to open a valve that allows the vacuum of space (above 100,000 feet) to pump on the liquid helium in the dewar and cool it down. In addition for the first mission a custom electronics box, the Capacitance Bridge Box will be used to make high-precision measurements of the pressure and density in one of the experiment cells.

Because of the ultra-high sensitivity of LTMPF's science cells and sensors the small amounts of heat left behind when a charged particle passes through these components represents a noise source; measurements from a charged-particle monitor can be correlated with the sensor-data noise to reduce their impact. In a similar fashion the accelerometer provided by the SAMS Project allows comparison of small vibrations with increases in signal noise. In this way Investigators are able to understand and mitigate a couple unwanted features of space experiments.

\section{Insert design and capabilities}

LTMPF provides to each Investigator a standard frame on which to build their cryogenic Insert. The assembled Insert for each science instrument is $20 \mathrm{~cm}$ diameter and $45 \mathrm{~cm}$ long. An Insert consists of a base plate (or Cold Plate) to which is mounted a thermo-mechanical truss (the Probe) which in turn supports the science cell and its actuators and sensors (the Instrument Sensor Package, or ISP). The science team integrates the unique components that they have developed in their laboratories on the standard Probe; they then attach the many wires and capillaries between the ISP and the Cold Plate required to control the experiment. The Cold Plate feeds those connections through a vacuum-tight interface into the Dewar vacuum space and ultimately to the outside of the dewar. The Probe interfaces the science instrument package to the dewar providing mechanical support and thermal isolation.

The thermal mechanical structure, or Probe, provides mechanical support and thermal isolation to the science instrument package. It consists of high strength and low thermal conductivity struts intercepted by thermal isolation stages made of high thermal conductivity material. It is designed to support $8 \mathrm{~kg}$ of mass during launch and provide enough thermal isolation for the inner most stage to be operating at sub-nano-Kelvin stability. Figure 2 shows a picture

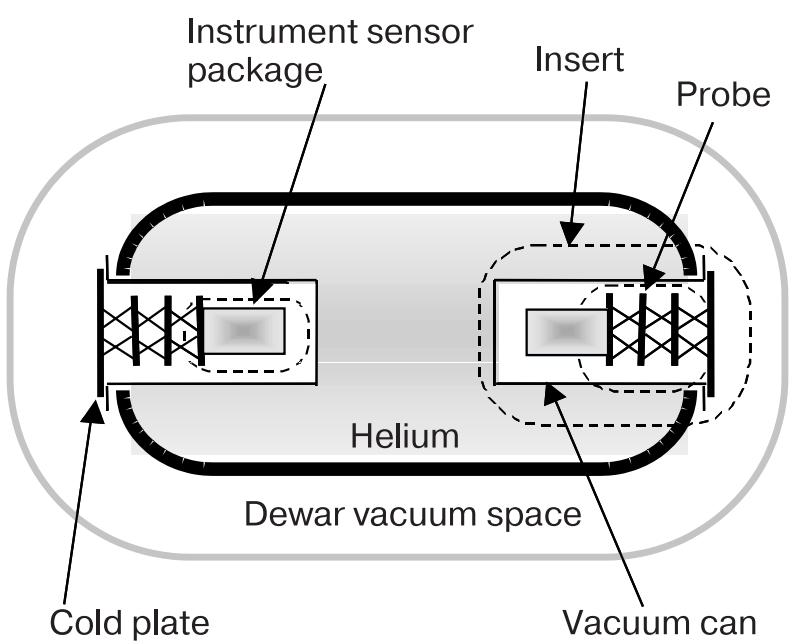

Fig. 2. Dewar interior.

of one such structure made of stainless steel struts and aluminum thermal isolation stages. In tests, this structure survived $7.7 \mathrm{~g}$ rms random vibration while supporting a $6.2 \mathrm{~kg}$ mass. Each thermal stage has also demonstrated an ability to intercept most of the heat variations from the previous stage, allowing only one part in 7000 to leak through to the next stage. With 3 stages, this is more than enough to allow thermal control to sub-nano-Kelvin stability.

The resonance frequency of the structure together with the supported mass is an important parameter in mitigating the launch-heating problem. It was successfully demonstrated by Cui et al. [5] that if this resonance frequency is sufficiently higher than the resonance frequency of the dewar, there is very little launch-heating. This implies that most of the heating arises from the flexing of the mechanical members of the instrument package. During testing, the structure shown in Fig. 3 and discussed above had a resonance of $72 \mathrm{~Hz}$ compared with the $48 \mathrm{~Hz}$ resonance expected

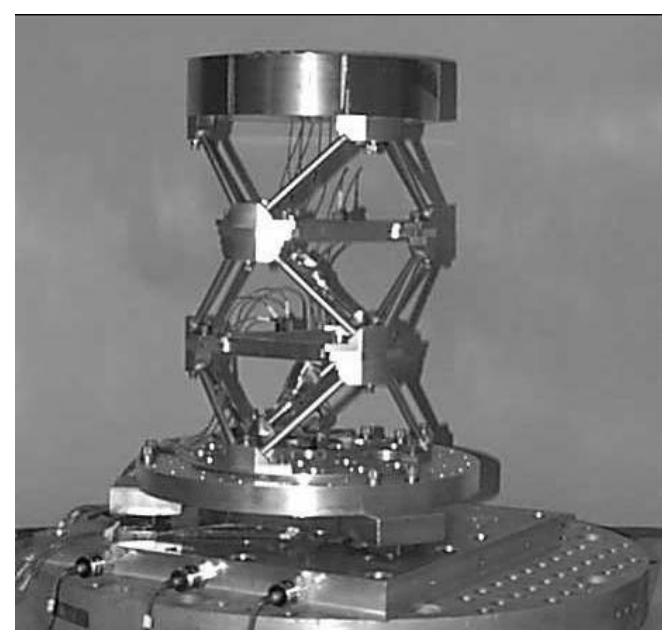

Fig. 3. A picture of the thermal mechanical structure. 
of the dewar. Thus we expect some mitigation of launch heating from this structure.

\section{Vacuum can and the adsorption pump}

A vacuum can maintains a high vacuum around each Insert for thermal isolation purposes. As long as the vacuum can is leak tight against the surrounding helium in the dewar, maintaining a high vacuum should not be a problem, since all gases except helium freeze. It has been learned in previous flights that the science instrument package can heat up to close to $10 \mathrm{~K}$ under the strong vibration of the launch if the Insert is launched in a vacuum. During these previous launches, it was necessary to fill the vacuum can with ${ }^{3} \mathrm{He}$-exchange gas to conduct the heat away from the science instrument to prevent it from heating up. Once on orbit, the exchange gas was vented to space and an adsorption pump, with activated charcoal, was used to absorb the rest of the gas. We inherited the adsorption pump design and modified it to make it lighter. The operation sequence is also inherited. The adsorption pump's design and operation are described in detail by Lysek et al. [6].

Due to their high sensitivity to magnetic fields, SQUIDs are usually operated inside a dewar with an external magnetic shield. This type of magnetic shield is heavy, weighing perhaps as much as $30 \mathrm{~kg}$. A way to reduce weight is to locate the shield outside the vacuum can instead. The shield is being developed and will be made of a special magnetic material Cryoperm 10 [7], which is known to have permeability of 50,000 at low temperatures. It is expected to weigh less than $6 \mathrm{~kg}$ and provides a magnetic environment with less than $10 \mathrm{mG}$ of field variation inside.

\section{Facility test, launch and operations}

The integration of the LTMPF payload, its instruments and the verification of the mechanical, thermal, power, telemetry, and command interfaces with the ISS is an integral part of the qualification process. This integration process starts at the Jet Propulsion Laboratory (JPL) and is completed at the launch site where the payload will be launched on a crossbay carrier in the shuttle following a successful Certification of Flight Readiness Review. The ISS Program will transport the LTMPF Facility Class Payload on an STS flight, perform all the payload accommodation and accommodation engineering, and all necessary transportation integration services.

Then the ISS Program and NASDA IP will install the LTMPF on ISS as an externally attached payload furnishing onorbit services to the payload for com- manding, telemetry and for ancillary data. The LTMPF payload after completing its 5 month onorbit mission life will be transferred from the ISS to an STS return flight for return of the payload to KSC. The payload will be returned to JPL for refurbishment and installation of two new instruments for the next mission.

\section{Conclusion}

The LTMPF currently under development will provide a unique environment of low temperature and microgravity for long duration. When the facility is ready for launch of the first mission in late 2005, it will open up exciting new science investigation opportunities onboard the International Space Station. JPL will provide the necessary infrastructure and services to enable a user-friendly interface to the scientific community, making easy and low cost access to space a reality for scientists.

\section{Acknowledgements}

This work is being carried out at the Jet Propulsion Laboratory, California Institute of Technology under contract to the National Aeronautics and Space Administration. The work was funded by NASA's Office of Biological and Physical Research, Physical Sciences Division. We thank BATC for providing the picture in Fig. 1.

1. T. Chui, A. Croonquist, M. Larson, F. Liu, W. Holmes, D. Langford, and J. Pensinger, AIAA J. 6, No 4, 4932 (2001).

2. P.V. Mason, D. Petrac, D.D. Elleman, T. Wang, H.W. Jackson, D.J. Collins, P. Cowgill, and J.R. Gatewood, Adv. Cryogenic Eng. 21, 869 (1985).

3. J.A. Lipa, D.R. Swanson, J.A. Nissen, T.C.P. Chui, and U.E. Israelsson, Phys. Rev. Lett. 76, 944 (1996).

4. J.A. Lipa, D.R. Swanson, J.A. Nissen, P.R. Williamson, Z.K. Geng, T.C.P. Chiu, U.E. Israelsson, and M. Larson, Phys. Rev. Lett. 84, 4894 (2000).

5. W. Cui, R. Almy, S. Deiker, D. McCammon, J. Morgenthaler, W.T. Sanders, R.L. Kelley, F.J. Marshall, S.H. Moseley, C.K. Stahle, and A.E. Szymkowiak, SPIE 2280, 362 (1994); D. McCammon, R. Almy, S. Deiker, J. Morgenthaler, R.L. Kelley, and F.J. Marshall, Nucl. Instrum. Methods A370, 266 (1996).

6. M.J. Lysek, U.E. Israelsson, T.C.P. Chui, M.E. Larson, D. Petrac, S.E. Elliott, D.R. Swanson, X. Qin, and J.A. Lipa, in: Adv. Cryogenic Eng. Proc. of the 16th International Cryogenic Engineering Conference, P. Kittel (ed.), Vol. 42, Portland, Oregon (1997).

7. Cryoperm 10, Amuneal Corp., 4737 Darrah. st., Philadelphia, PA 19124-2705. 